\title{
Consentement À Payer Et Rentabilité D'une Innovation En Agro-Alimentaire : Cas Du Décorticage Mécanique Et De La Fortification En Fer Du Sorgho Dans Le Nord-Bénin
}

\author{
Morest M. T. Agossadou \\ Sandrine S. Sêgla \\ Anselme A. Adégbidi \\ Université d'Abomey-Calavi, Faculté des Sciences Agronomiques, \\ Département d'Economie, Sociologie, Anthropologie et Communication \\ pour le Développement Rural \\ Polycarpe A. P. Kayodé \\ Université d'Abomey-Calavi, Faculté des Sciences Agronomiques, \\ Département de Nutrition et Sciences Alimentaires
}

Doi: 10.19044/esj.2018.v14n21p155 URL:http://dx.doi.org/10.19044/esj.2018.v14n21p155

\begin{abstract}
The purpose of this study is twofold. Firstly, it is a question of assessing the determinants of the willingness to pay (WTP) of farming households in Thian, a village located in northern Benin, exposed to the risk of anemia, to benefit from an agro-food innovation aimed at reduce these risks : dehulling and iron fortification of sorghum. Secondly, there was talk of evaluating the financial profitability of this innovation. To do this, socioeconomic and financial data were collected from 90 chef-kitchens prepared to adopt the innovation, the operator in charge of shelling and fortification services, the supplier of shelling equipment and the supplier of iron. The contingent valuation method was used to collect respondents' WTP and the linear regression model to identify the determinants of these WTP and calculate their mean value. In addition, the cash flow method was used to assess the financial profitability of de-hulling and fortification services. The results reveal that the WTP (whose average is 24.4 FCFA) is negatively influenced by the fact that the respondent belongs to the pilot phase of the project and the average quantity of sorghum devoted to the consumption of dibou (sorghum paste). In a context where the monetary discount rate is $12 \%$, mechanical shelling and iron fortification of sorghum as an economic activity is not profitable.
\end{abstract}


Keywords: Willingness to pay, financial profitability, agri-food innovation, NPV, sorghum

\section{Resume}

L'objectif de la présente étude est double. Primo, il s'agit d'évaluer les déterminants du consentement à payer (CAP) des ménages agricoles de Thian, village situé au nord-bénin, exposés à des risques d'anémie, pour bénéficier d'une innovation agro-alimentaire destinée à réduire ces risques : le décorticage et la fortification en fer du sorgho. Deuxièmement, il a été question d'évaluer la rentabilité financière de cette innovation. Pour ce faire, des données socio-économiques et financières ont été collectées auprès de 90 chef-cuisines prédisposées à adopter l'innovation, de l'opérateur en charge des services de décorticage et de fortification, du fournisseur des équipements de décorticage et du fournisseur du fer. La méthode d'évaluation contingente a été utilisée pour recueillir les CAP des enquêtées et le modèle de régression linéaire pour identifier les déterminants de ces CAP et calculer leur valeur moyenne. Par ailleurs, il a été fait usage de la méthode des cash-flows pour apprécier la rentabilité financière des services de décorticage et de fortification. Les résultats révèlent que le CAP (dont la moyenne est de 24,4 FCFA) est influencé négativement par l'appartenance de l'enquêtée à la phase pilote du projet et la quantité moyenne de sorgho consacrée à la consommation de dibou (pâte de sorgho). Dans un contexte où le taux d'actualisation monétaire est de $12 \%$, le décorticage mécanique et la fortification en fer du sorgho, comme activité économique, n'est pas rentable.

Mots clés : Consentement à payer, rentabilité financière, innovation agroalimentaire, VAN, sorgho

\section{Introduction}

La carence en fer touche 4 à 5 milliards de personnes soit 66 à $80 \%$ de la population mondiale (OMS, 2003). L'anémie quant à elle affecte environ 2 milliards de personnes (soit $30 \%$ de la population mondiale). D'après les chiffres de l'OMS, l'anémie en Afrique toucherait 45 millions d'enfants de moins de 5 ans, 58 millions de femmes en âge de procréer et 11 millions de femmes enceintes. En Afrique sub-saharienne, la carence en fer est la principale cause d'anémie. Cette forme d'anémie, causée par la carence en fer, est désignée sous le vocable d'anémie martiale (ou ferriprive). Les femmes en âge de procréer et les enfants constituent les groupes les plus vulnérables. Les conséquences de cette maladie sur ces groupes vulnérables sont énormes. L'anémie ferriprive provoque des dégâts irréversibles au niveau du cerveau, une diminution de la réponse immunitaire et donc une augmentation de la fréquence des infections chez les enfants en bas âge. Chez l'adulte, elle 
provoque de la fatigue et une capacité au travail réduite. Plus de naissances prématurées et d'enfants mort-nés sont les dégâts de ce mal chez la femme enceinte (Franziska, 2000).

La carence en fer est principalement liée au fait que le fer alimentaire absorbé ne permet pas de couvrir les besoins élevés des populations à risques (Berger et Dillon, 2002 ; Franziska, 2000). Ceci est dû soit à la consommation d'aliments pauvres en fer, soit à la non ou faible disponibilité du fer contenu dans les aliments consommés. Pour faire face à ce problème, diverses stratégies existent comportant chacune des avantages et des inconvénients (Dillon, 2000). Il s'agit de la diversification alimentaire, de la supplémentation en fer, des mesures de santé publique et de l'enrichissement (ou fortification) en fer des aliments (Inwent, 2006 ; Alaoui, 2005 ; Berger et Dillon, 2002; Dillon, 2000). Dans les pays en développement, la fortification est de plus en plus, souvent, reconnue comme une approche efficace, à moyen et à long termes pour améliorer l'état en micronutriments de larges couches de la population (Inwent, 2006). Elle est considérée comme l'un des moyens les moins coûteux pour surmonter la malnutrition en micronutriments (Banque mondiale, 1994 ; Unicef, 1998). Elle consiste à ajouter du fer dans un aliment de consommation courante, afin d'augmenter le niveau de consommation de ce nutriment par la population (Inwent, 2006 ; Berger et Dillon, 2002). L'aliment qui transporte le nutriment est appelé véhicule ou vecteur.

$\mathrm{Au}$ Bénin, selon les statistiques de l'EDSB-III (2006), l'anémie ferriprive touche respectivement $78 \%$ et $61 \%$ d'enfants de 6 à 59 mois et de mères de 15 à 49 ans. Quatre vingt deux pour cent (82\%) des enfants anémiés se situent en milieu rural, contre $70 \%$ en milieu urbain. Chez les enfants, les taux d'anémie ferriprive les plus élevés se retrouvent dans les départements de 1'Alibori $(89,7 \%)$, de la Donga $(88,1 \%)$ et les plus faibles dans les départements du Littoral $(60,3 \%)$ et du Borgou $(69,6 \%)$. Chez les mères, les taux d'anémie ferriprive sont les plus élevés dans les départements de l'Ouémé (75\%), l'Alibori (67\%), l'Atlantique (65\%), le Plateau (63\%) et le Mono $(61 \%)$. Les taux les plus faibles se retrouvent dans les autres départements et sont compris entre $50 \%$ et $57 \%$. Ces statistiques montrent que l'anémie ferriprive est un réel problème de santé publique au Bénin. Des actions concrètes doivent, de ce fait, être menées pour l'éradication de ce mal. Cet objectif ne peut être atteint sans l'identification des causes réelles de cette forme d'anémie dans ces milieux touchés. Il faut donc identifier le repas le plus consommé, voir sa composition chimique (notamment en fer) et évaluer la biodisponibilité de ce fer afin de savoir si l'anémie ferriprive, dans ces milieux, est liée à l'insuffisance de fer dans les repas consommés ou à la non disponibilité pour l'organisme du fer contenu dans les aliments consommés. Dans le premier cas, une fortification serait suffisante, mais dans le second, une amélioration de la biodisponibilité sera plus raisonnable. 
Dans cette optique, une étude a été conduite dans le nord-Bénin par Kayodé et al (2005). Cette étude a révélé que le sorgho est une céréale qui occupe une place importante dans les habitudes alimentaires des populations concernées. Cette place importante qu'occupe cette céréale est imputable à la diversité d'aliments qui en sont issus, et à leur importance respective dans l'alimentation des ménages. Il s'agit des pâtes (dibou, sifanou, foura), des bouillies (koko, sorou, kamanguia) et des boissons (tchoukoutou, chakpalo). Selon ces mêmes auteurs, seulement 2 à $6 \%$ des $4 \mathrm{mg} / 100 \mathrm{~g}$ du fer contenu dans le sorgho est disponible aux consommateurs de ces types d'aliments. Ce faible niveau de disponibilité du fer est dû à son inhibition par les facteurs antinutritionnels (FAN), c'est-à-dire les tannins, le calcium/phosphore, les phytates et fibres et les oxalates, qui sont principalement localisés dans le péricarpe des graines (Kayodé et Denou, 2007). A partir de ce moment, la cause de l'anémie ferriprive dans le nord du Bénin n'est pas une insuffisance de fer dans les repas consommés, mais plutôt une faible disponibilité du fer contenu dans les aliments consommés.

Pour améliorer la biodisponibilité du fer contenu dans le sorgho, il convient, logiquement, de réduire, voir supprimer, les facteurs antinutritionnels qui y sont contenus et ce, par l'enlèvement du péricarpe des graines.

Il y a quelques décennies, les consommateurs du sorgho se livraient chaque jour à un travail manuel d'enlèvement du péricarpe et de pulvérisation des grains avant de pouvoir préparer le repas quotidien (Bassey et Schmidt, 1990). Les difficultés inhérentes à ces activités ont conduit ces consommateurs à désormais pulvériser directement les grains (de sorgho) avec l'apparition du moulin. A partir de ce fait, il ne serait plus raisonnable de proposer à ces consommateurs de sorgho d'enlever manuellement (ou traditionnellement) le péricarpe des grains. Il faut donc penser à une technologie pouvant jouer ce rôle. Mais l'enlèvement du péricarpe du sorgho, dans le but de réduire ou éliminer les facteurs antinutritionnels, n'est pas sans conséquences.

En effet, les $4 \mathrm{mg} / 100 \mathrm{~g}$ de fer contenu dans le grain de sorgho se localisent au même endroit que les facteurs antinutritionnels, i.e. dans le péricarpe. La réduction ou l'élimination des facteurs antinutritionnels par enlèvement du péricarpe entraine alors une réduction ou une élimination du fer contenu dans le sorgho. Pour ne pas revenir à la situation de départ où les consommateurs du sorgho entier présentent une carence en fer, un enrichissement des graines ou de la farine doit être effectué après l'enlèvement du péricarpe.

Ainsi, par le biais du projet intitulé 'Introduction of a mechanical dehuller and iron fortification in the traditional processing of sorghum in Benin to improve the iron status of rural consumers of porridge" initié par le Centre Régional de Nutrition et d'Alimentation Appliquées (CERNA) de la 
Faculté des Sciences Agronomiques de l'Université d'Abomey-Calavi, une plate-forme de décorticage mécanique et de fortification en fer du sorgho est installée dans Thian, un village de la commune de Parakou, département du Borgou. Le décorticage ou l'enlèvement du péricarpe se fait de façon mécanique à l'aide d'un décortiqueur de type Engelbert et la fortification en fer est manuelle.

Le caractère nouveau de cette plate-forme et des services qu'elle offre fait d'elle une innovation. Une phase d'expérimentation de l'innovation a été effectuée entre octobre 2009 et juin 2010 dans le but d'évaluer son adaptation aux réalités alimentaires du village. Pour cette raison, vingt-quatre ménages contenant au moins un individu anémié ont été retenus pour décortiquer mécaniquement et fortifier en fer gratuitement le sorgho avant de préparer le repas. Avant le démarrage de la phase d'exécution proprement dite du projet, au cours de laquelle les services offerts par la plate-forme seront désormais payants et son accès sera donné à toute la population, quelques questions s'étaient posées : tous les ménages du village accepteront-ils décortiquer mécaniquement et fortifier leur sorgho avant de préparer le repas, dans le but d'améliorer leur statut en fer ? accepteront-ils payer pour le décorticage mécanique et la fortification en fer du sorgho ? quels montants sont-ils prêts à payer pour? quels sont les facteurs qui influencent cette décision de décortiquer, fortifier le sorgho et payer pour cela ? Quels sont les facteurs qui influencent le montant que les ménages se proposent de payer? Ces montants suffiront-ils pour couvrir les charges de fonctionnement de la plate-forme?

Dans un autre article, les questions relatives à l'acceptation de l'innovation et les déterminants y afférents ont été élucidées. Le but du présent papier est d'évaluer le consentement à payer (CAP) des ménages pour bénéficier de l'innovation, le décorticage et la fortification en fer du sorgho, puis d'évaluer sa rentabilité financière.

\section{Matériel et méthodes de collecte des données}

L'étude s'est déroulée en 2010 dans le nord-Bénin, département du Borgou, commune de Parakou, et plus précisément dans le village Thian (Figure 1). Il s'agit du village retenu dans le cadre du projet, pour la phase d'expérimentation de l'innovation. Le décorticage mécanique se fait à l'aide d'un décortiqueur de type Engelbert fabriqué localement par le Centre de Recherche en Technologie Alimentaire et la fortification, à l'aide du NaFeEDTA fourni sous le nom commercial 'Ferrazone' par la société Akzo Nobel. 


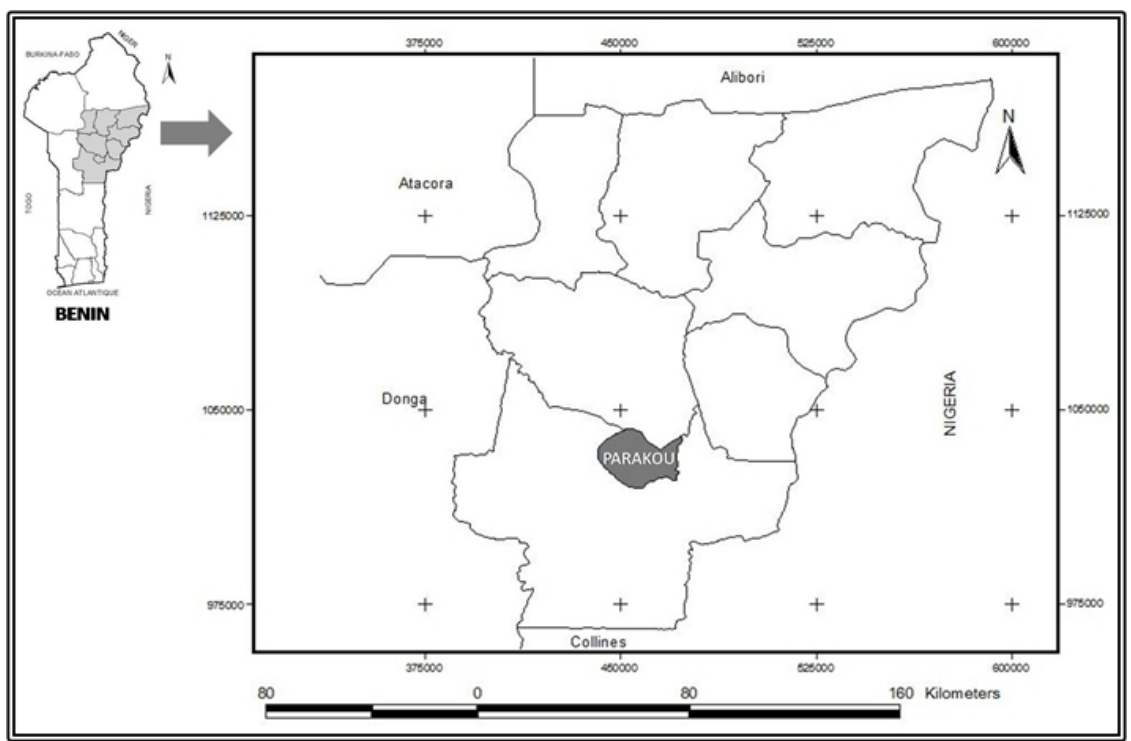

Figure 1. Carte du département du Borgou mentionnant la commune de Parakou.

Le mode d'échantillonnage aléatoire stratifié a été utilisé pour sélectionner 120 ménages "consommateurs" de dibou. Le critère de stratification est la participation ou non à la phase d'expérimentation de l'innovation ; deux strates ont été obtenues : les ménages "'consommateurs" de dibou retenus pour la phase d'expérimentation de l'innovation (strate 1) et les ménages "consommateurs" de dibou non retenus pour la phase d'expérimentation (strate 2). Les effectifs des strates 1 et 2 sont respectivement de 24 et 118 ménages. En utilisant la méthode de proportionnalité, respectivement 20 et 100 ménages ont été aléatoirement tirés des strates 1 et 2 pour faire un total de 120 ménages comme échantillon de notre étude. Chaque ménage était représenté par la femme à charge de la préparation du dibou (chef-cuisine).

\section{Méthode d'évaluation du CAP}

Les données d'évaluation du CAP ont été collectées chez 90 chefcuisines (parmi les 120) qui ont déclaré leur disposition à adopter l'innovation, c'est-à-dire prêtes à payer une somme d'argent pour en bénéficier. Dans le cadre de cette étude, il s'agit de recueillir la valeur monétaire que les chefcuisines prédisposées à adoptées l'innovation confère à celle-ci. Deux grandes méthodes sont généralement utilisées à cette fin : la méthode des préférences révélées et la méthode des préférences déclarées (méthode d'évaluation contingente).

L'approche des préférences révélées présente une perception ex-post des préférences et se fonde sur l'observation des comportements des agents économiques (Gniglo, 2010). Elle n'est pas compatible à notre cas d'étude vu 
que le projet n'est pas encore entré dans sa phase active où les services de décorticage et de fortification sont payés.

Par contre, l'évaluation contingente, quant à elle, est conçue dans le but d'amener les individus à déclarer leurs préférences, afin de donner une valeur à des biens publics qui n'ont pas de prix sur le marché (Maresca et al, 2006). Elle consiste à collecter des informations sur les préférences des consommateurs, en leur demandant ce qu'ils consentiraient à payer pour un changement de dotation en bien ou service non-marchand, ou bien la compensation minimum qu'ils exigeraient si le changement n'a pas lieu (Weber, 2003 ; Yelkouni, 2004 ; Gbinlo, 2010). Elle a beaucoup servi dans le domaine de l'environnement (gestion des déchets, amélioration de la qualité de l'air, dommages causés par des marées noires, etc.) qui est d'ailleurs son premier champ d'application. Elle a été utilisée par Ouedraogo et al. (2007) dans la gestion des risques en agriculture urbaine irriguée, notamment pour évaluer le consentement à payer pour une amélioration de la qualité de l'eau pour le maraîchage dans les villes de Ouagadougou et de Bobo-Dioulasso au Burkina-Faso. En 2007, Monsi Agboka a fait usage de la même méthode pour évaluer la valeur économique des plantes utilisées pour les soins gynécologiques dans les terroirs autour de la Réserve de Biosphère de la Pendjari au Bénin. En 2010, cette méthode a été utilisée par l'Agence de l'Eau Rhin-Meuse dans le cadre d'une étude coûts-bénéfices de l'amélioration de la qualité d'un cours d'eau dans la région de la Bouvade en France.

Cette méthode s'est révélée incontournable plus tard dans beaucoup de pays, et dans beaucoup d'autres domaines que l'environnement, notamment dans les domaines de l'alimentation (Blanchemanche et al. (2009)), de la santé (Le Goff et Nassiri (2005)), de la télécommunication, etc. bien qu'elle expose le chercheur (s'il n'y prend garde) à des biais tels que le biais stratégique, le biais de l'information et le biais hypothétique. Tout ceci a amené à retenir cette méthode pour déterminer la valeur que les ménages "consommateurs" de dibou accordent à une amélioration nutritionnelle de leur repas. Grace à des modèles ou combinaisons de modèles économétriques, le consentement à payer moyen se détermine et les facteurs susceptibles de l'influencer aussi. Le choix du modèle ou de la combinaison de modèles économétriques dépend du mode de déclaration de la valeur appliqué.

Terra (2005) identifie cinq (5) modes de déclaration de la valeur : la question ouverte, la question fermée, la double question fermée, le système d'enchères et la carte de payement. Ces différents modes de révélation avec leurs caractéristiques respectives sont présentés dans le tableau 1.

Tableau 1: Caractéristiques des différents modes de révélation de la valeur.

\begin{tabular}{l|c|c|l|l}
\hline Méthode & $\begin{array}{l}\text { Incitation à la } \\
\text { révélation }\end{array}$ & Effort cognitif & $\begin{array}{l}\text { Risque } \\
\text { d'ancrage }\end{array}$ & $\begin{array}{l}\text { Taille d'échantillon } \\
\text { (relative) requise }\end{array}$ \\
\hline Question ouverte & Faible & Elevé & Aucun & La plus faible \\
\hline
\end{tabular}




\begin{tabular}{l|c|l|l|l}
\hline Question fermée & Très élevée & Très faible & -- & La plus élevée \\
\hline $\begin{array}{l}\text { Double question } \\
\text { Fermée }\end{array}$ & -- & Modéré & Elevée \\
\hline Système d'enchères & Faible & Modéré & Elevé & Modérée \\
\hline $\begin{array}{l}\text { Carte de } \\
\text { paiement }\end{array}$ & Elevée & Modéré & -- & Faible \\
\hline
\end{tabular}

Source : Terra, 2005

Note : ( -- ) est mis pour les cellules où l'information n'est pas connue.

D'après l'analyse de ce tableau, la question ouverte nous semble plus adéquate et sera donc retenue pour l'évaluation monétaire du bien qui est la farine issue de sorgho décortiqué et fortifié avec du fer, vu le niveau d'effort cognitif, le niveau du risque d'ancrage et la taille de l'échantillon requise. Le faible niveau d'incitation observé chez les enquêtés est dû au fait que la plupart des enquêtes qui ont conduit à la réalisation de ce tableau sont faites par téléphone, e-mail ou la poste. Nous espérons donc que ce niveau d'incitation à la révélation sera stimulé avec notre moyen d'enquête qui est direct (enquêté face à enquêteur).

Lorsque l'option est faite d'utiliser la question ouverte comme mode de révélation de la valeur du bien, Terra (2005) recommande l'utilisation du modèle de Heckman (ensemble modèle Probit et régression linéaire). Le modèle de Heckman a été appliqué par Miwoto et al. (2017) afin d'identifier les déterminants du consentement des ménages pour une gestion durable d'une forêt au sud-ouest du Bénin. Gbinlo (2010) Miwoto et al. (Ibid) précisent le caractère séquentiel de ce modèle qui est constitué de l'équation de sélection (Probit) répondant à la question « êtes-vous prête à décortiquer et fortifier votre sorgho et surtout payer une somme d'argent pour bénéficier de ces services ? » et de l'équation substantielle (régression linéaire) répondant à la question «si oui à la question précédente, combien seriez-vous prête à payer ? ». La première séquence a déjà été prise en compte dans le cadre d'un autre article. Dans le présent, seule la deuxième séquence a valeur d'être. En conséquence, le choix a porté sur un modèle de régression linéaire par la méthode des moindres carrés pour identifier les déterminants du consentement à payer les services de décorticage mécanique et de fortification en fer du sorgho par les chef-cuisines et calculer le consentement à payer moyen. Ce modèle se présente comme suit :

$$
\mathrm{y}_{\mathrm{i}}=\beta_{0}+\sum_{\mathrm{k}=1}^{\mathrm{K}-1} \beta_{\mathrm{k}} \mathrm{x}_{\mathrm{ki}}+\varepsilon_{\mathrm{i}} \quad \mathrm{i}=1,2, \ldots, \mathrm{n} ;
$$

Avec :

$K=$ nombre de variables explicatives

$y_{i}=$ variable dépendante ou de réponse $;$ ici c'est le CAP pour décortiquer et fortifier $1,85 \mathrm{~kg}$ de sorgho

$x_{k i}=$ kème variable explicative ou régresseur 
$\varepsilon_{\mathrm{i}}=$ résidu (ou erreur non observée)

L'estimation de ce modèle est basée sur la méthode des moindres carrés ordinaires (MCO). Elle a été faite grâce au logiciel STATA.

\section{Spécification du modèle}

Le modèle empirique peut s'écrire :

$$
\begin{aligned}
\text { CAP }_{\mathrm{i}}=\beta_{0}+ & \beta_{1} \text { AGE }+\beta_{2} \text { REVENU }+\beta_{3} \text { PERSCHARG }+\beta_{4} \text { APPP } \\
& +\beta_{5} \text { ANEMIE }+\beta_{6} \text { QUANT }+\beta_{7} \text { ACMARI }+\beta_{8} \text { STATU }+\varepsilon_{i}
\end{aligned}
$$

Avec,

$\mathrm{CAP}_{\mathrm{i}}=$ consentement à payer de l'individu $\mathrm{i}$

$\beta_{\mathrm{i}}=$ coefficient du régresseur $\mathrm{i}$

$\varepsilon_{\mathrm{i}}=$ résidu

AGE : variable désignant l'âge de l'enquêtée. On s'attend à un effet négatif. En effet, plus le répondant prend de l'âge, moins elle est apte à se reproduire puisque proche de la ménopause ou déjà en ménopause. De plus, dans ces conditions, la probabilité est faible qu'elle ait à sa charge des enfants de moins de 5 ans. Sachant que les enfants de moins de 5 ans et les femmes enceintes sont la principale cible de l'anémie ferriprive, on pourrait donc s'attendre à ce que plus l'âge des chef-cuisine'" augmente, plus leurs CAP seront faibles.

REVENU : désigne le revenu de l'enquêtée. Il est attendu un signe positif pour cette variable, car plus le revenu de l'enquêtée est élevé, plus elle pourra en utiliser pour améliorer la qualité nutritionnelle de son repas.

PERSCHARG : désigne le nombre total de personnes à charge de l'enquêtée. Il est attendu un effet négatif. En effet, plus le répondant a de personnes à sa charge, plus il aura d'individus à nourrir et plus grandes seront les dépenses du ménage. N'ayant donc plus assez d'excédents de ressources, son consentement à payer est susceptible de diminuer.

APPP : cette variable désigne l'appartenance ou non de l'enquêtée à la phase pilote du projet. Il est attendu un signe positif car nous présumons que les répondants ayant participé à la phase pilote ont une bonne connaissance des risques d'anémie ferriprive liés à la consommation du dibou issu de sorgho non décortiqué et ont également une bonne connaissance des attributs de l'unité de décorticage et de fortification. Leurs consentements à payer devrait être plus élevés.

ANEMIE : indique le passé du ménage par rapport à l'anémie. L'idée ici est que les ménages ayant au moins un membre qui a déjà souffert de l'anémie seront prêts à donner 'tout' ce qu'ils peuvent pour ne plus côtoyer ce mal car 
les dépenses liées à son traitement seraient outrancières. Nous nous attendons donc à un signe positif.

QUANT : représente la quantité moyenne de sorgho que le ménage consacre à la consommation de dibou. Un effet négatif est attendu. En effet, plus grande est la quantité de sorgho moulu chaque fois que l'enquêtée va au moulin, plus élevé seront les charges liées à la mouture. Elle ne sera donc plus en mesure de consacrer un montant assez important pour le décorticage et la fortification. Son consentement à payer sera donc " petit' .

APMARI : désigne la disposition du mari de l'enquêtée à l'appuyer. Cet appui est soit financier (augmentation de l'argent de popote) soit en nature (augmentation de la quantité de sorgho). Un effet positif de cette variable est attendu. En effet, le décorticage du sorgho réduit la quantité de farine et par conséquent diminue soit le nombre de jours que doit faire le contenu du grenier du ménage, soit le niveau de satisfaction. Pour faire face à ces difficultés, les ménages seront obligés d'augmenter la contenance de leurs greniers. Les chefcuisines qui bénéficieront de l'appui de leur mari dans ce sens auront un consentement à payer élevé.

STATU : statut social de l'enquêtée après l'adoption de la technologie. Un effet positif de cette variable sur le CAP est attendu lorsque l'enquêtée perçois la technologie comme pouvant améliorer son statut social.

La nature des variables, leurs codes, modalités et les signes attendus sont résumés dans le tableau 2. Le tableau 3 présente Hypothèses à vérifier, problèmes liés à leur violation et méthodes/tests utilisées pour leur détection.

Tableau 2 : Noms, types, codes, modalités et signes attendus des coefficients des variables du modèle de régression linéaire multiple.

\begin{tabular}{|c|c|c|c|c|}
\hline Nom de la variable & Type & Code & Modalités & $\begin{array}{l}\text { Signe } \\
\text { attendu }\end{array}$ \\
\hline \multicolumn{5}{|l|}{ Variable de réponse } \\
\hline $\begin{array}{l}\text { Consentement à payer de } \\
\text { l'enquêtée }\end{array}$ & Continu & CAP & l & I \\
\hline \multicolumn{5}{|l|}{ Régresseurs } \\
\hline Age de l'enquêtée. & Continu & AGE & l & - \\
\hline Revenu de l'enquêtée. & Continu & REVENU & I & + \\
\hline $\begin{array}{l}\text { Nombre de personnes à } \\
\text { charge }\end{array}$ & Continu & PERSCHARG & I & - \\
\hline $\begin{array}{l}\text { Passé du ménage par } \\
\text { rapport à l'anémie. }\end{array}$ & Binaire & ANEMIE & $\begin{array}{l}0=\text { jamais d'anémié dans } \\
\text { le ménage } ; 1=\text { au moins } \\
\text { un membre du ménage } \\
\text { déjà anémié. }\end{array}$ & + \\
\hline
\end{tabular}




\begin{tabular}{l|l|l|l|c}
\hline $\begin{array}{l}\text { Quantité moyenne de } \\
\text { sorgho consacrée à la } \\
\text { consommation de dibou. }\end{array}$ & Continu & QUANT & $/$ & - \\
\hline $\begin{array}{l}\text { Appartenance de } \\
\text { l'enquêtée à la phase } \\
\text { pilote du projet. }\end{array}$ & Binaire & APPP & $\begin{array}{l}0=\text { n'a pas appartenu; 1= } \\
\text { a appartenu }\end{array}$ & + \\
\hline $\begin{array}{l}\text { statut social de l'enquêtée } \\
\text { après l'adoption de la } \\
\text { technologie }\end{array}$ & Binaire & STATU & $\begin{array}{l}0=\text { pas d'amélioration ; } \\
1=\text { amélioration. }\end{array}$ & + \\
\hline $\begin{array}{l}\text { Disposition du mari de } \\
\text { l'enquêtée à l'appuyer }\end{array}$ & Binaire & APMARI & $\begin{array}{l}0=\text { pas disposé ; 1= } \\
\text { disposé }\end{array}$ & + \\
\hline
\end{tabular}

Note $:(/)=$ pas d'informations.

Tableau 3 : Hypothèses à vérifier, problèmes liés à leur violation et méthodes/tests utilisées pour leur détection.

\begin{tabular}{l|l|c}
\hline \multicolumn{1}{c|}{ Hypothèses colinéarité des } & Multicolinéarité & Méthodes/tests de détection \\
\hline $\begin{array}{l}\text { Non } \\
\text { régresseurs }\end{array}$ & $\begin{array}{l}\text { Analyse de la matrice de } \\
\text { corrélation des régresseurs. }\end{array}$ \\
\hline $\begin{array}{l}\text { Spécification « correcte »du } \\
\text { modèle }\end{array}$ & $\begin{array}{l}\text { Erreur (ou biais) de spécification du } \\
\text { modèle }\end{array}$ & Test RESET de Ramsey \\
\hline
\end{tabular}

\section{Méthode d'évaluation de la rentabilité financière}

Plusieurs méthodes existent pour l'évaluation (ex-ante) de la rentabilité financière. Il s'agit entre autre de la méthode de trésorerie prévisionnelle, la méthode d'établissement des comptes d'exploitation générale prévisionnels et la méthode des cash-flows.

La méthode de trésorerie prévisionnelle consiste à prévoir période par période, les recettes totales et les dépenses totales liées à l'exercice d'une activité donnée. La différence entre recettes totales et dépenses totales permet de prévoir la situation de la trésorerie en fin de chaque période. La limite de cette méthode est qu'elle ne prend pas en compte les amortissements. Elle ne s'intéresse donc pas au renouvellement des biens immobilisés. Elle ne sera pas utilisée dans le cadre de notre étude.

L'établissement des comptes d'exploitation générale prévisionnels consiste à prévoir période par période les produits totaux et les charges totales liés à l'exercice d'une activité donnée. La différence entre produits totaux et charges totales permet de calculer les résultats de l'activité à la fin de chaque période. Cette méthode prend en compte les amortissements, mais ne prévoit pas de la liquidité pour leur renouvellement. C'est en cela que réside sa limite. Elle ne sera donc pas utilisée dans le cadre de notre travail.

La méthode des cash-flows est semblable aux deux précédentes. Elle prévoit également période par période, les coûts totaux et les bénéfices totaux liés à l'exercice d'une activité donnée : on parle d'analyse coûts-bénéfices. La différence entre cette méthode et les deux autres est qu'elle tient compte 
automatiquement de l'amortissement puisqu'elle prévoit une année zéro au cours de laquelle les biens immobilisés sont acquis, le renouvellement des immobilisations en fonction de leur durée de vie et l'inscription de leurs valeurs résiduelles dans les bénéfices à la fin de l'exercice. Elle prévoit également en année zéro le capital de fonctionnement (ou fond de roulement) qui est la somme totale nécessaire à l'autofinancement quotidien de l'exécution de l'activité. Cette méthode des cash-flows vient combler les lacunes présentées par les autres. Elle est donc celle qui a été retenue et utilisée dans le cadre de cette étude.

ECOFIN (2004) propose quatre critères pour déterminer la durée de l'analyse financière d'un projet. Parmi ces critères, figure la durée de vie du point de vue technique/économique des biens les plus coûteux du projet. Dans notre cas, l'analyse a été effectuée sur une période équivalente à la durée de vie de la décortiqueuse (10 ans) qui est le bien le plus coûteux. Les coûts d'existence et de fonctionnement et les bénéfices de la plate-forme ont été ensuite calculés par année. Les cash-flows ont été également calculés par année suivant la formule :

\section{Cash - flows $=$ Total bénéfices - Total côuts}

Les coûts regroupent les dépenses d'investissements fixes, le capital de fonctionnement et les dépenses courantes. Les dépenses d'investissement fixes sont les dépenses consenties pour acquérir les immobilisations. Elles regroupent, les dépenses effectuées pour l'achat du décortiqueur et le crépissage du planché du local qui l'abrite. Le capital de fonctionnement, désigne la somme totale nécessaire à l'autofinancement quotidien de l'exécution de l'activité. Il a été calculé à partir de l'estimation des coûts et bénéfices liés au fonctionnement quotidien de l'activité. Les dépenses courantes sont la somme nécessaire au financement quotidien de l'exécution de l'activité pendant une année. Elles concernent la rémunération du minotier et le loyer qui lui est payé pour la location de son moteur, les frais d'entretien du décortiqueur, les dépenses d'achat d'une boite vide de tomate qui servira d'unité de mesure du sorgho à décortiquer, les dépenses d'achat de 7,2 kg tous les ans du produit Ferrazone pour la fortification et le transport du produit Ferrazone depuis la société de fabrication, localisée aux Pays-Bas, jusqu'à Thian. Les bénéfices, quant à eux, prennent en compte les entrées d'argent liées aux activités de décorticage et de fortification. Ces entrées sont calculées à partir de l'estimation de la quantité totale de sorgho à décortiquer par les ménages prédisposés (extrapolée à l'univers de l'échantillonnage) au cours d'une année, et du consentement à payer moyen des chef-cuisine enquêtées. Les bénéfices prennent également en compte les valeurs résiduelles des immobilisations et la valeur résiduelle du capital de fonctionnement. Ces données financières ont été collectées auprès de l'opérateur en charge des 
services de décorticage et de fortification, du fournisseur des équipements de décorticage et du fournisseur du fer synthétique.

En nous servant des cash-flows par années, nous avons calculé la Valeur Actuelle Nette (VAN) du profit généré par l'investissement et le Taux de Rentabilité Interne (TRI) de l'activité. La VAN a été calculée après application d'un taux d'actualisation de la monnaie égal à $12 \%$ correspondant au taux commercial généralement utilisé au Bénin. Si elle est supérieure à zéro, cette activité est considérée comme financièrement rentable. Il faut préciser que cette évaluation de rentabilité s'est basée sur l'hypothèse de stationnarité des coûts et des bénéfices durant la période d'analyse. On parle de scénario «normal». Lorsque le scénario «normal» se révèle favorable (VAN positive), on passe à au moins un scénario « pessimiste » (baisse des bénéfices et/ou augmentation des coûts), puis à au moins un scénario «optimiste » (baisse des coûts et/ou augmentation des bénéfices) pour voir la réaction de l'activité. Mais, lorsque le scénario «normal» est défavorable (VAN négative), on ne prévoit que des scénarii « optimistes ». Cette analyse qui consiste à prévoir des scénarii lors d'une analyse de rentabilité financière est appelée analyse de sensibilité. Elle a été réalisée dans le cadre de cette étude.

\section{Résultats et discussions}

\section{Déterminants du CAP des ménages consommateurs de dibou Présentation des résultats}

Les tableaux 4 et 5 présentent respectivement les corrélations entre les variables explicatives et les résultats de l'estimation du modèle de régression linéaire.

Tableau 4 : Matrice de corrélation entre les variables explicatives du modèle de régression linéaire.

\begin{tabular}{|c|c|c|c|c|c|c|c|c|}
\hline & AGE & REVENU & PERSCHARG & ANEMIE & QUANT & APPP & STATU & APMARI \\
\hline AGE & 1,0000 & & & & & & & \\
\hline REVENU & 0,0334 & 1,0000 & & & & & & \\
\hline PERSCHARG & 0,0556 & 0,1426 & 1,0000 & & & & & \\
\hline ANEMIE & 0,1104 & 0,0508 & $0,2174 *$ & 1,0000 & & & & \\
\hline QUANT & $\begin{array}{l}- \\
0,0104\end{array}$ & 0,0598 & $-0,0294$ & $-0,1243$ & 1,0000 & & & \\
\hline APPP & 0,1002 & $0,1564 *$ & $0,1618 *$ & $-0,0000$ & $-0,0362$ & 1,0000 & & \\
\hline STATU & - & 0,0449 & 0,0032 & 0,1256 & $-0,0689$ & 0,1180 & 1,0000 & \\
\hline APMARI & $\begin{array}{l}- \\
0,0368\end{array}$ & $0,1543 *$ & 0,0979 & $-0,0953$ & 0,0230 & $\begin{array}{l}- \\
0,0149\end{array}$ & $0,2581^{*}$ & 1,0000 \\
\hline
\end{tabular}


Le coefficient de corrélation le plus élevé $(0,2581)$ est significatif au seuil de $10 \%$. Cette valeur du coefficient de corrélation le plus élevé s'est révélé « faible » car inférieur à 0,8 ; la multicolinéarité n' est, de ce fait, pas un sérieux problème.

Tableau 5 : Résultats de la régression linéaire multiple.

\begin{tabular}{|c|c|c|c|c|c|}
\hline Variables & $\begin{array}{l}\text { Signes } \\
\text { attendus }\end{array}$ & Coefficients & $\begin{array}{l}\text { Erreurs standards } \\
\text { robusts }\end{array}$ & $\begin{array}{l}\text { Statistiques } \mathrm{t} \text { de } \\
\text { Student }\end{array}$ & Significativité \\
\hline Constante & 1 & 22,1097 & 8,151705 & 2,71 & $0,008 * * *$ \\
\hline $\begin{array}{l}\text { Quantité moyenne de } \\
\text { sorgho consacrée à la } \\
\text { consommation } \\
\text { (QUANT). }\end{array}$ & - & $-0,9860733$ & 0,4017155 & $-2,45$ & $0,016 * *$ \\
\hline $\begin{array}{l}\text { Appartenance } r \text { de } \\
\text { l'enquêtée à la phase } \\
\text { pilote du projet (APPP). }\end{array}$ & + & $-13,95706$ & 2,772587 & $-5,03$ & $0,0001 * * *$ \\
\hline \multicolumn{6}{|c|}{$\begin{array}{l}\mathrm{R}^{2}=0,183 \\
\text { Prob }>\mathrm{F}=0,0005 * * * \\
\text { Nombre d'observations }=90\end{array}$} \\
\hline
\end{tabular}

\section{- Signification globale du modèle}

La p-value du modèle est sensiblement nulle (inférieure à $1 \%$ ); cela signifie que l'on prend un risque de se tromper de moins de $1 \%$ en concluant que les variables explicatives sélectionnées apportent une quantité d'information significative au modèle. Le modèle est donc globalement significatif au seuil de $1 \%$.

\section{- Qualité de l'ajustement du modèle}

Le coefficient de détermination multiple $\mathrm{R}^{2}$ du modèle est égal à 0,183 ; cela signifie que $18,3 \%$ des variations du consentement à payer sont expliquées par les régresseurs du modèle. Au plan statistique, ce coefficient de détermination est faible. Toutefois, Gujarati (2004) avait relevé que dans les études en coupe instantanée comprenant plusieurs observations, obtient généralement un faible $\mathrm{R}^{2}$, en raison de la diversité des unités dans ce type d'études. Une telle valeur du $\mathrm{R}^{2}$ est donc acceptable.

\section{- Les variables déterminantes et le consentement à payer moyen}

Les variables qui influencent le consentement à payer des chefs-cuisine pour les opérations de décorticage mécanique et de fortification en fer du sorgho sont : l'appartenance de l'enquêtée à la phase pilote du projet (APPP) et la quantité moyenne de sorgho consacrée à la consommation (QUANT).

Le CAP moyen, estimé à partir du modèle économétrique, est de 24,4 FCFA pour 1,85 kg de sorgho décortiqué et fortifié en fer.

\section{- Spécification « correcte » du modèle.}

Le tableau 6 présente les résultats du test RESTE de Ramsey. 
Tableau 6 : Résultats du test RESET de Ramsey.

\begin{tabular}{c}
\hline Ramsey RESET test using powers of the fitted values of cap \\
\hline Ho: aucune variable pertinente n'est omise \\
\hline $\mathrm{F}(3,84)=0,86$ \\
Prob $>\mathrm{F}=0,4673$
\end{tabular}

La probabilité du test est 0,4673 . L'hypothèse Ho ne saurait être rejetée au seuil de $10 \%$. Aucune variable explicative importante n'a donc été omise dans la spécification du modèle ; ce qui témoigne de la bonne spécification du modèle.

\section{Discussions}

Influence de la variable «quantité moyenne de sorgho consacrée à la consommation de dibou » sur le CAP

Pour rappel, la variable «quantité moyenne de sorgho consacrée à la consommation de dibou» désigne la quantité moyenne de sorgho que l'enquêtée emmène à la minoterie, chaque fois qu'elle est dans le besoin de farine pour préparer la pâte. Elle a une influence négative sur le consentement à payer des chef-cuisines. Ce résultat correspond aux prévisions. Il indique que plus grande est la quantité moyenne de sorgho consacrée à la consommation de dibou, plus faible est le consentement à payer pour décortiquer et fortifier cette quantité de sorgho. En effet, le prix de la mouture d'une boite de tomate de sorgho non décortiqué $(1,85 \mathrm{~kg})$ dans le village Thian est de 75 FCFA. Il est imposé à tous les minotiers par leur association professionnelle, et s'applique donc dans toutes les minoteries. Pour les chefcuisines enquêtées, en fonction de leurs contraintes budgétaires, ce prix de mouture est perçu comme élevé. N'ayant pas de meilleurs choix, elles se trouvent dans l'obligation de s'y conformer. En conséquence, les ménages à effectif élevé et qui consacrent une quantité élevée de sorgho à la consommation de dibou dépenseront davantage dans la mouture. Ces dépenses incompressibles relatives à la mouture du sorgho, ajoutées à celles liées à son décorticage et sa fortification se révèleront davantage plus élevées pour ces ménages. Et puisqu'ils ne peuvent pas choisir l'option de réduire la quantité moyenne de sorgho consacrée à la consommation de dibou au risque de créer un déséquilibre alimentaire au niveau quantitatif, ils ont préféré diminuer le montant qu'ils désirent payer pour le décorticage et la fortification. A préférences sensorielles et nutritionnelles, et niveau d'information donnés, le consommateur est contraint dans ses choix par le budget dont il dispose, c'està-dire son revenu et les prix auxquels il fait face (Etiévant et $a l, 2010)$. D'où, l'influence négative de la variable 'quantité moyenne de sorgho consacrée à la consommation de dibou" sur le consentement à payer. 
Au-delà de l'explication relative à la contrainte budgétaire, le choix opéré par les chef-cuisines d'accorder une moindre valeur monétaire à l'amélioration nutritionnelle du repas de base de leur ménage trouve ses fondements théoriques en économie. En effet, selon la théorie économique de la maximisation de l'utilité, les ménages et les individus ne prennent la décision d'adopter, d'acheter un bien ou service que lorsqu'ils y trouvent une utilité supplémentaire (Adesina et Seidi, 1995; Adesina, 1996; Rahm et Huffman, 1984). Ainsi, dans le processus mental décisionnel, l'individu va estimer ses avantages à adopter, acheter le bien ou service, puis les comparer aux coûts qu'il devra engager. Dans le domaine de l'alimentation, ces avantages et coûts dépendent du plaisir hédonique immédiat de l'ingestion, de l'adhésion aux normes sociales d'alimentation en vigueur, de la valeur accordée à la santé et du prix nécessaire à l'obtention du repas (Etiévant et $a l$, Ibid). Lorsqu'il perçoit ce bilan économique et social négatif pour lui, il s'abstient d'aller vers le bien ou le service. Dans le cas de présenté dans cet article, probablement, les chef-cuisines n'ont pas perçu ou n'ont pas su apprécier à sa juste valeur l'utilité qu'elles et leurs familles tireraient de l'adoption du décorticage mécanique et de la fortification en fer du sorgho. Cela pose une fois de plus la problématique de rationalité dans les choix inhérente à la théorie de la maximisation de l'utilité.

\section{L'appartenance de l'enquêtée à la phase pilote du projet}

Contrairement aux prévisions, cette variable présente une influence négative sur le montant que les enquêtées consentent payer, pour bénéficier d'une farine de sorgho améliorée. Autrement dit, lorsqu'on va des enquêtées n'ayant pas appartenu à la phase pilote du projet à celles ayant appartenue à cette phase du projet, la valeur accordée à la farine de sorgho améliorée diminue. Ce résultat, bien que contraire aux attentes, s'explique.

En effet, pour avoir participé à la phase d'essai du projet, certaines chef-cuisines ont eu l'occasion de se confronter aux limites relatives à l'innovation : absence répétée de l'agent qui effectue la fortification, faiblesse de la durée de conservation de la farine et la diminution de la quantité de farine après le décorticage. Les déceptions occasionnées par ses limites expliquent la faible motivation des chef-cuisines pour l'innovation matérialisée par la faiblesse de la valeur monétaire qu'elles y accordent. Par ailleurs, le fait qu'elles aient été habituées à un service de décorticage et de fortification gratuit pourrait justifier leur faible intérêt à payer un montant important pour bénéficier d'une farine nutritionnellement meilleure. Ce comportement des chef-cuisines, déjà habituées à avoir les services de décorticage et de fortification gratuitement, face à la décision de payer désormais les mêmes services trouve ses fondements théoriques dans le domaine du marketing. En effet, la phase du pilote du projet avec le principe d'accès gratuit aux services 
peut être considérée en marketing comme une phase de promotion des ventes. La promotion des ventes se définit comme un ensemble de techniques utilisées exceptionnellement dans la vie d'un produit (ou d'un service) aux fins de développer à court terme les ventes tout en conciliant l'intérêt du consommateur et le profit de l'entreprise (Troadec, 1975). Parmi ces techniques de vente figure les promotions par les prix dont une des variantes les plus populaires est la réduction temporaire du prix du produit ou du service (Parsons, 2003). Pour Engel et al (1995), l'efficacité de cette technique est à double tranchant. Pendant qu'elle contribue à une augmentation des ventes pendant et après le temps de la promotion (Chandon, 1997), la promotion par la réduction des prix peut affaiblir la loyauté des clients vis-à-vis du produit ou du service. Ils resteront fidèles seulement jusqu'à la fin de la promotion ou jusqu'à ce qu'un produit concurrent offre une baisse de prix plus importante (Engel, Ibid). Cette théorie explique parfaitement le consentement à payer des chef-cuisines ayant participé à la phase pilote du projet (phase de promotion) où le prix des services de décorticage mécanique et de fortification en fer du sorgho était réduit à la valeur nulle.

Rentabilité financière de l'activité de décorticage mécanique et de fortification en fer du sorgho.

Présentation des résultats et discussions

Le tableau 7 présente les résultats de l'analyse de rentabilité financière. 
Année

\section{COUTS}

Achat du décortiqueur

Crépissage du planché du local abritant le décortiqueur

\section{Total Investissements}

Fond de roulement

Rémunération du minotier+location moteur

Entretien décortiqueur

Achat d'une boite vide de tomate

Gaz-oil

Achat d'une bassine

Achat du produit Ferrazone pour la fortification

Transport du produit Ferrazone (7,2kg)

\section{Totale dépenses courantes}

Total coûts

\section{BENEFICES}

Décorticage sorgho

Valeur résiduelle du décortiqueur

Valeur résiduelle du planché

Valeur résiduelle du fond de roulement

Total bénéfices

Cach flow $=$ Total bénéfices - Total coûts

$$
\begin{aligned}
& \text { VAN } \\
& \text { TRI }
\end{aligned}
$$

\begin{tabular}{|c|c|c|c|c|c|c|c|c|c|c|}
\hline 20000 & & & & & 20000 & & & & & \\
\hline 370000 & 0 & 0 & 0 & 0 & 20000 & 0 & 0 & $\mathbf{0}$ & $\mathbf{0}$ & $\mathbf{0}$ \\
\hline \multicolumn{11}{|l|}{891272,4} \\
\hline & 120000 & 120000 & 120000 & 120000 & 120000 & 120000 & 120000 & 120000 & 120000 & 120000 \\
\hline 0 & 10000 & 10000 & 10000 & 10000 & 10000 & 10000 & 10000 & 10000 & 10000 & 10000 \\
\hline 0 & 300 & 300 & 300 & 300 & 300 & 300 & 300 & 300 & 300 & 300 \\
\hline 0 & 81180 & 81180 & 81180 & 81180 & 81180 & 81180 & 81180 & 81180 & 81180 & 81180 \\
\hline 0 & 1000 & 1000 & 1000 & 1000 & 1000 & 1000 & 1000 & 1000 & 1000 & 1000 \\
\hline 0 & 30700,8 & 30700,8 & 30700,8 & 30700,8 & 30700,8 & 30700,8 & 30700,8 & 30700,8 & 30700,8 & 30700,8 \\
\hline 0 & 53910 & 53910 & 53910 & 53910 & 53910 & 53910 & 53910 & 53910 & 53910 & 53910 \\
\hline 0 & 297090,8 & 297090,8 & 297090,8 & 297090,8 & 297090,8 & 297090,8 & 297090,8 & 297090,8 & 297090,8 & 297090,8 \\
\hline 1261272,4 & 297090,8 & 297090,8 & 297090,8 & 297090,8 & 317090,8 & 297090,8 & 297090,8 & 297090,8 & 297090,8 & 297090,8 \\
\hline 0 & 432345 & 432345 & 432345 & 432345 & 432345 & 432345 & 432345 & 432345 & 432345 & 432345 \\
\hline 0 & & & & & & & & & & 0 \\
\hline 0 & & & & & & & & & & 0 \\
\hline 0 & & & & & & & & & & 891272,4 \\
\hline 0 & 432345 & 432345 & 432345 & 432345 & 432345 & 432345 & 432345 & 432345 & 432345 & 1323617,4 \\
\hline-1261272 & 135254,2 & 135254,2 & 135254,2 & 135254,2 & 115254,2 & 135254,2 & 135254,2 & 135254,2 & 135254,2 & 1026526,6 \\
\hline
\end{tabular}

350000

Tableau 7 : Analyse de rentabilité financière de l'activité de décorticage mécanique et de fortification en fer du sorgho. 
De ce tableau, il ressort que la VAN du profit généré par l'investissement, après application d'un taux d'actualisation égal à $12 \%$, est inférieure à zéro. Ceci témoigne de ce que l'activité de décorticage mécanique et de fortification en fer du sorgho n'est pas financièrement rentable. Cependant, le taux de rentabilité interne égal à $9 \%$ renseigne qu'en absence de toute autre perturbation dans l'environnement économique, l'activité de décorticage mécanique et de fortification en fer du sorgho serait financièrement rentable (VAN supérieure à zéro) lorsqu'on applique au capital investi un taux d'intérêt inférieur à $9 \%$.

Le scénario «normal » étant défavorable, notre analyse de sensibilité a porté sur trois scénarii optimistes :

- $\quad$ scénario 1 : la demande des services de décorticage mécanique et de la fortification en fer du sorgho augmente de $5 \%$ tous les ans ;

- $\quad$ Scénario 2 : la demande des services de décorticage mécanique et de la fortification en fer du sorgho augmente de $10 \%$ tous les ans ;

- $\quad$ scénario 3 : le transport du produit Ferrazone est assuré par une ONG, le gouvernement ou une organisation multinationale.

Le tableau 8 présente la réaction de l'activité dans chaque cas.

Tableau 8 : Réaction de l'activité de décorticage et de fortification en fer du sorgho dans trois situations différentes.

\begin{tabular}{ccc}
\hline Scénarii & VAN & TRI \\
\hline Scénario 1 & 157223,77 & $14 \%$ \\
Scénario 2 & 787515,48 & $25 \%$ \\
Scénario 3 & 74254,32 & $13 \%$ \\
\hline
\end{tabular}

Ce tableau montre que, lorsque la demande des services de décorticage mécanique et de fortification en fer du sorgho augmente de $5 \%$ ou de $10 \%$ tous les ans, l'activité est financièrement rentable. Il en est de même, lorsque les frais de transport du produit Ferrazone sont assurés par une ONG, le gouvernement ou une organisation multinationale.

A travers les résultats des analyses de sensibilité, on se rend compte que les défis à relever pour assurer la rentabilité des services de décorticage et de fortification sont l'accroissement des ventes, donc du volume de sorgho décortiqué par unité de temps, et la réduction des coûts liés à l'offre de services. Cette augmentation du chiffre d'affaire peut se faire par des actions d'éducation nutritionnelle des ménages du village, tout en s'accentuant sur les risques afférents à la consommation de repas à base de sorgho non décortiqué, bien que pas toujours immédiatement visibles. L'éducation nutritionnelle se définit comme un ensemble d' activités de communication visant la 
modification volontaire des pratiques qui ont une incidence sur l' état nutritionnel de la population, dans la perspective d'une amélioration de celuici (Müller et Krawinkel, 2005). La réduction des coûts, en dehors de la subvention des coûts de transport par une tierce organisation, peut s'opérer en substituant le carburant que nécessite la décortiqueuse pour son fonctionnement (le gaz-oil vendu à 550 FCFA le litre) par l'huile de Jatropha curcas (325 FCFA le litre). Dans les mêmes conditions, ce biocarburant ferait tourner le moteur pendant environ le double de l'unité de temps pendant laquelle le gaz-oil le fait tourner. De plus, il contribuerait à un meilleur entretien du moteur que le gaz-oil.

\section{Conclusion}

La présente étude a révélé que le consentement à payer des ménages du village de Thian pour bénéficier du décorticage mécanique et de la fortification en fer du sorgho en vue de réduire le risque d'anémie ferriprive auquel ils sont exposés, est influencé négativement par deux variables : la quantité moyenne de sorgho consacrée à la consommation du repas et l'appartenance de l'enquêtée à la phase pilote du projet.

Par ailleurs, l'analyse de rentabilité financière effectuée a montré que l'opération de décorticage et de fortification en fer, en scénario normal, n'est pas financièrement rentable. Il convient donc de travailler à réaliser les scénarios optimistes proposés en sensibilisant les ménages et en réduisant les coûts relatifs à l'offre des services.

\section{References:}

1. Adesina A A, 1996. Factors affecting the adoption of fertilizers by rice farmers in Côte d'Ivoire: Nutrient cycling. Agroecosystems 46, 29-39.

2. Adesina A A, Seidi S, 1995. Farmers' perceptions and adoption of new agricultural technology: Analysis of modern mangrove rice varieties in Guinea Bissau. Quarterly Journal of International Agriculture 34, 35-85.

3. Blanchemanche S, Treich N \& Tello R, 2009. Evaluation socioéconomique en appui à la gestion des risques alimentaires. Volet I Méthodes d'évaluation socio-économique appliquées aux risques alimentaires. Rapport d'activité. MAP \& INRA. 69 p.

4. Chandon P, 1997. Promotion des ventes, psychologie de l'achat et comportement de consommation. Thèse pour le doctorat en sciences de gestion. Ecole des Hautes Etudes Commerciales. Jouy-en-Josas, France.

5. ECOFIN, 2004. Analyse Financière et Economique des Projets. Syllabus Cours de Base. 
6. Engel J F, Blackwell R D, Miniard P W, 1995. Consumer behavior, $8^{\mathrm{e}}$ ed. Coll. The Dryden Press Series in marketing. Fort Worth; Montréal : Dryden Press, 951 p.

7. Etiévant P, Bellisle F, Dallongeville J, Etilé F, Guichard E, Padilla M, Romon-Rousseaux M, (éditeurs), 2010. Les comportements alimentaires. Quels en sont les déterminants ? Quelles actions, pour quels effets ? Expertise scientifique collective, rapport, INRA (France), 275 p.

8. Gbinlo R, 2010. Organisation et financement de la gestion des déchets ménagers dans les villes de l'Afrique Subsaharienne : le cas de la ville de Cotonou au Bénin. Thèse pour le doctorat en sciences économiques. Université d'Orléans. France.

9. Gujarati D, 2004. Econométrie. Traduction de la 4è édition américaine par B. Bernier.

10. Kayodé APP, Adégbidi A, Linnemann AR, Nout MJR, \& Hounhouigan JD, 2007. Perception par les consommateurs de la qualité des variétés de sorgho paysannes en usage au Bénin et de leurs produits dérivés. Ecology of Food and Nutrition 44, 271-294.

11. Le Goff-Pronost M, \& Nassiri N, 2005. Deux approches nouvelles dans l'évaluation de la télémédecine : 1'évaluation contingente et l'analyse multicritère. Cahier de recherche, $\mathrm{N}^{\circ} 7$.

12. Maresca B, Ranvier M \& Dujin A, 2006. Valoriser l'action publique : Le "consentement à payer", un outil au service de la LOLF. Cahier de recherche $\mathrm{n}^{\circ}$ 224. CREDOC.

13. Monsi Agboka F, 2007. Estimation de la valeur économique des biens 'non commercialisés' : cas des plantes utilisées pour soins gynécologiques dans les terroirs autour de la Réserve de Biosphère de la Pendjari. Mémoire d'ingénieur agronome FSA/UAC. AbomeyCalavi.

14. Müller O, Krawinkel M, 2005. Malnutrition and health in developing countries. CMAJ $2005 ; 173: 279-86$.

15. Ouedraogo D, Sangaré D, Tougma A T, 2007. Gestion des risques en agriculture urbaine irriguée et consentement à payer pour une amélioration de la qualité de l'eau pour le maraîchage dans les villes de Ouagadougou et de Bobo-Dioulasso au Burkina. Université polytechnique de Bobo-Dioulasso (UPB). Institut du développement rural (IDR). Burkina-Faso.

16. Parsons A G, 2003. Assessing the effectiveness of shopping mall promotions: customers analyis. International journal of retail \& distribution management, vol 31, $\mathrm{n}^{\circ} 2-3, \mathrm{p} 74$. 
17. Rahm M R, Huffman W E, 1984. The Adoption of Reduced Tillage: The Role of Human Capital and Other Variables. American Journal of Agricultural Economics 66, 405-413.

18. Terra S, 2005. Guide de bonnes pratiques pour la mise en œuvre de la méthode d'évaluation contingente. Paris : Ministère de l'écologie et du développement durable, $83 \mathrm{p}$.

19. Troadec L, 1975. Manuel de promotion des ventes. Coll. Manuels EO/formation permanente/formation permanente. Paris : Editions d'Organisation, $238 \mathrm{p}$.

20. Weber J, 2003. L'évaluation contingente : les valeurs ont-elles un prix ? http://www.ceri-sciences-po.org.

21. Yelkouni M, 2004. Gestion d'une ressource naturelle et action collective : le cas de la forêt de Tiogo au Burkina Faso. Thèse pour le doctorat en sciences économiques. Université d'Auvergne-Clermont I. France. 